\title{
Darboux integrability and invariant algebraic curves for planar polynomial systems
}

\author{
Colin Christopher ${ }^{1}$, Jaume Llibre ${ }^{2}$, Chara Pantazi ${ }^{2}$ and Xiang Zhang ${ }^{3}$ \\ ${ }^{1}$ Department of Mathematics and Statistics, University of Plymouth, Plymouth PL2 3AJ, UK \\ 2 Department de Matemàtiques, Universitat Autònoma de Barcelona, 08193-Bellaterra, \\ Barcelona, Spain \\ ${ }^{3}$ Department of Mathematics, Shanghai Jiaotong University, Shanghai 200030, \\ People's Republic of China \\ E-mail: C.Christopher@plymouth.ac.uk, jllibre@mat.uab.es, hpant@mat.uab.es \\ and m_x_zhang@263.net
}

Received 15 June 2001, in final form 12 October 2001

Published 1 March 2002

Online at stacks.iop.org/JPhysA/35/2457

\begin{abstract}
In this paper we study the normal forms of polynomial systems having a set of given generic invariant algebraic curves.

PACS numbers: $02.30 . I k, 02.10$. De
\end{abstract}

\section{Introduction and statement of the main results}

Nonlinear ordinary differential equations appear in many branches of applied mathematics and physics. In this paper we only consider autonomous differential systems. For a twodimensional system the existence of a first integral completely determines its phase portrait. Of course, the easiest planar integrable systems are the Hamiltonian ones. The planar integrable systems which are not Hamiltonian can be in general very difficult to detect. Many different methods have been used for studying the existence of first integrals for non-Hamiltonian systems based on: Noether symmetries [5], the Darboux theory of integrability [14], the Lie symmetries [7, 23], the Painlevé analysis [2], the use of Lax pairs [19], the direct method $[16,17]$, the linear compatibility analysis method [27], the Carlemann embedding procedure $[1,6]$, the quasimonomial formalism [3], etc.

The algebraic theory of integrability is a classical one, which is related to the first part of the Hilbert's 16th problem. This kind of integrability is usually called Darboux integrability, and it provides a link between the integrability of polynomial systems and the number of invariant algebraic curves they have (see Darboux [14] and Poincaré [24]).

Jouanolou [18] extended the planar Darboux theory of integrability to polynomial systems in $\mathbb{R}^{n}$ or $\mathbb{C}^{n}$, for extension to other fields see [28]. In $[4,9,10,20]$, the authors developed the Darboux theory of integrability essentially in $\mathbb{R}^{2}$ or $\mathbb{C}^{2}$ considering not only the invariant 
algebraic curves but also the exponential factors, the independent singular points and the multiplicity of the invariant algebraic curves.

Prelle and Singer [25], using methods of differential algebra, showed that if a polynomial vector field has an elementary first integral, then it can be computed using the Darboux theory of integrability. Singer [26] proved that if a polynomial vector field has Liouvillian first integrals, then it has integrating factors given by Darbouxian functions. Some related results can be found in [8].

In this paper we are mainly interested in the polynomial differential systems which have a given set of invariant algebraic curves, independent of whether they are integrable or not. Thus, first we study the normal forms of planar polynomial vector fields having a given set of generic invariant algebraic curves. That is, in some sense we are interested in a kind of inverse theory of the Darboux theory of integrability.

In this work we deal with the following planar (differentiable) polynomial system of degree $m$ :

$$
\dot{x}=P(x, y) \quad \dot{y}=Q(x, y)
$$

where $P, Q \in \mathbb{C}_{m}[x, y]$, the set of complex polynomials of degree at most $m$ in the variables $x$ and $y$, and $\max \{\operatorname{deg} P, \operatorname{deg} Q\}=m$.

Let $C(x, y) \in \mathbb{C}[x, y]$, the ring of complex polynomials in $x$ and $y$. The algebraic curve $C(x, y)=0$ of $\mathbb{C}^{2}$ is called an invariant algebraic curve of system (1) if

$$
P C_{x}+Q C_{y}=K C
$$

for some complex polynomial $K(x, y)$, which is called the cofactor of $C=0$. Here and after, we denote by $C_{x}$ and $C_{y}$ the derivatives of $C$ with respect to $x$ and $y$, respectively. For simplicity, in what follows we will talk about the curve $C=0$, only saying the curve $C$.

We mention that the cofactor of the invariant algebraic curve for system (1) has degree at most $m-1$. Let $C=\prod_{i=1}^{l} C_{i}^{n_{i}}$ be the irreducible decomposition of $C$. Then $C$ is an invariant algebraic curve with a cofactor $K$ of system (1) if and only if $C_{i}$ is an invariant algebraic curve with a cofactor $K_{i}$ of system (1). Moreover, we have $K=\sum_{i=1}^{l} n_{i} K_{i}$. For a proof see [13].

Let $F(x, y)=\exp (G(x, y) / H(x, y))$ with $G, H \in \mathbb{C}[x, y]$ coprime (or equivalently, $(G, H)=1)$. We say that $F$ is an exponential factor if

$$
P F_{x}+Q F_{y}=L F
$$

for some polynomial $L \in \mathbb{C}_{m-1}[x, y]$, which is called a cofactor of $F$.

Let $U$ be an open subset of $\mathbb{C}^{2}$. A complex function $\mathcal{H}: U \rightarrow \mathbb{C}$ is a first integral of system (1), if it is constant on all solution curves $(x(t), y(t))$ of system (1), i.e., $\mathcal{H}(x(t), y(t)) \equiv$ constant for all values of $t$ for which the solution $(x(t), y(t))$ is defined on $U$. If the first integral $\mathcal{H}$ is differentiable, then $P \mathcal{H}_{x}+Q \mathcal{H}_{y}=0$ in $U$.

If there exists a smooth function $\mathcal{R}(x, y)$ such that $(P \mathcal{R})_{x}+(Q \mathcal{R})_{y}=0$, then $\mathcal{R}$ is called an integrating factor of system (1).

If system (1) has a first integral or an integrating factor of the form

$$
C_{1}^{\lambda_{1}} \cdots C_{p}^{\lambda_{p}} F_{1}^{\mu_{1}} \cdots F_{q}^{\mu_{q}}
$$

where $C_{i}$ and $F_{j}$ are the invariant algebraic curve and exponential factor of system (1) respectively and $\lambda_{i}, \mu_{j} \in \mathbb{C}$, then system (1) is called Darboux integrable. This kind of function is called a Darbouxian function.

Our first result is the following theorem.

Theorem 1. Let $C_{i}=0$ for $i=1, \ldots, p$, be irreducible invariant algebraic curves in $\mathbb{C}^{2}$, and set $r=\sum_{i=1}^{p} \operatorname{deg} C_{i}$. We assume that all $C_{i}$ satisfy the following generic conditions: 
(i) There are no points at which $C_{i}$ and its first derivatives all vanish.

(ii) The highest order terms of $C_{i}$ have no repeated factors.

(iii) If two curves intersect at a point in the finite plane, they are transversal at this point.

(iv) There are no more than two curves $C_{i}=0$ meeting at any point in the finite plane.

(v) There are no two curves having a common factor in the highest order terms.

Then any polynomial vector field $\boldsymbol{X}$ of degree $m$ tangent to all $C_{i}=0$ satisfies one of the following statements.

(a) If $r<m+1$ then

$$
\boldsymbol{X}=\left(\prod_{i=1}^{p} C_{i}\right) \boldsymbol{Y}+\sum_{i=1}^{p} h_{i}\left(\prod_{\substack{j=1 \\ j \neq i}}^{p} C_{j}\right) \boldsymbol{X}_{C_{i}}
$$

where $\boldsymbol{X}_{C_{i}}=\left(-C_{i y}, C_{i x}\right)$ is a Hamiltonian vector field, the $h_{i}$ are polynomials of degree no more than $m-r+1$, and the $\boldsymbol{Y}$ is a polynomial vector field of degree no more than $m-r$.

(b) If $r=m+1$ then

$$
\boldsymbol{X}=\sum_{i=1}^{p} \alpha_{i}\left(\prod_{\substack{j=1 \\ j \neq i}}^{p} C_{j}\right) \boldsymbol{X}_{C_{i}}
$$

with $\alpha_{i} \in \mathbb{C}$.

(c) If $r>m+1$ then $\boldsymbol{X}=0$.

This theorem, attributed to Christopher [10], was stated in several papers without proof such as $[10,12]$, and used in other papers $[4,21]$. The proof that we present here of theorem 1 circulated as the preprint [11] but was never published. Zholadek in [29] (see also theorem 3 of [30]) stated a similar result to our theorem 1, but as far as we know the paper [29] has not been published. In any case Zholadek's approach to theorem 1 is analytical, while our approach is completely algebraic.

Statement (b) of this theorem has a corollary proposed by Christopher and Kooij [12] showing that system (6) has the integrating factor $\mathcal{R}=\left(\prod_{i=1}^{p} C_{i}\right)^{-1}$, and consequently the system is Darboux integrable.

The following result shows that the generic conditions of theorem 1 are necessary.

Theorem 2. If one of the conditions (i)-(v) of theorem 1 is not satisfied, then the statements of theorem 1 do not hold.

We have mentioned that system (1) satisfying the five assumptions of theorem 1 with $r=m+1$ are Darboux integrable. Now we provide two examples of polynomial systems satisfying all assumptions of theorem 1 with $r=m+1$ except either (ii) or (iii) and which are not Darboux integrable. Until now there are very few proofs of polynomial systems which are not Darboux integrable, see for instance Jouanolou [18] and Maciejewski et al [22].

Consider the following quadratic systems:

$$
\begin{aligned}
& \dot{x}=y(a x-b y+b)+x^{2}+y^{2}-1 \\
& \dot{y}=b x(y-1)+a\left(y^{2}-1\right)
\end{aligned}
$$


which have the invariant circle $C_{1}=x^{2}+y^{2}-1=0$ with cofactor $K_{1}=2(x+a y)$ and the invariant straight line $C_{2}=y-1=0$ with cofactor $K_{2}=b x+a y+a$. We note that $C_{1}$ and $C_{2}$ are tangent at the point $(0,1)$.

Theorem 3. There are values of the parameters a and b for which system (7) is not Darboux integrable.

As a corollary the following result shows that there are polynomial systems with an invariant algebraic curve whose highest order term has repeated factors such that they are not Darboux integrable. Consider the following quadratic system:

$$
\begin{aligned}
& \dot{x}=(1-b)\left(x^{2}+2 y-1\right)-(a x-b)(y-1)=P(x, y) \\
& \dot{y}=-(b x+2 a y-a)(y-1)=Q(x, y)
\end{aligned}
$$

which has the invariant algebraic curves $C_{1}=x^{2}+2 y-1=0$ with cofactor $K_{1}=$ $2[(1-b) x-a y+a]$ and $C_{2}=y-1=0$ with cofactor $K_{2}=-(b x+2 a y-a)$. We note that the highest order term of $C_{1}$ has a repeated factor $x$.

Corollary 4. There exist values of the parameters a and b for which system (8) is not Darboux integrable.

The paper is organized as follows. In sections 2 and 3 we prove theorems 1 and 2, respectively. The proofs of theorem 3 and corollary 4 are given in sections 4 and 5 , respectively.

\section{Proof of theorem 1.}

In the proof of this theorem we will use intensively Hilbert's nullstellensatz (see, for instance, [15]):

Set $A, B_{i} \in \mathbb{C}[x, y]$ for $i=1, \ldots, r$. If $A$ vanishes in $\mathbb{C}^{2}$ whenever the polynomials $B_{i}$ vanish simultaneously, then there exist polynomials $M_{i} \in \mathbb{C}[x, y]$ and a non-negative integer $n$ such that $A^{n}=\sum_{i=1}^{r} M_{i} B_{i}$. In particular, if all $B_{i}$ have no common zero, then there exist polynomials $M_{i}$ such that $\sum_{i=1}^{r} M_{i} B_{i}=1$.

In what follows if we have a polynomial $A$ we will denotes its degree by $a$. If we do not say anything we denote by $C^{c}$ the homogeneous part of degree $c$ for the polynomial $C$. We shall need the following result.

Lemma 5. If $C^{c}$ has no repeated factors, then $\left(C_{x}, C_{y}\right)=1$.

Proof. Suppose that $\left(C_{x}, C_{y}\right) \neq 1$. Then there exists a polynomial $A$ nonconstant such that $A \mid C_{x}$ and $A \mid C_{y}$. Here $A \mid C_{x}$ means that the polynomial $A$ divides the polynomial $C_{x}$. Therefore, $A^{a} \mid\left(C^{c}\right)_{x}$ and $A^{a} \mid\left(C^{c}\right)_{y}$. By the Euler theorem for homogeneous polynomials we have that $x\left(C^{c}\right)_{x}+y\left(C^{c}\right)_{y}=c C^{c}$. So $A^{a} \mid C^{c}$. Since $A^{a},\left(C^{c}\right)_{x},\left(C^{c}\right)_{y}$ and $C^{c}$ are homogeneous polynomials of $\mathbb{C}[x, y]$ and $A^{a}$ divides $\left(C^{c}\right)_{x},\left(C^{c}\right)_{y}$ and $C^{c}$, the linear factors of $A^{a}$ having multiplicity $m$, must be linear factors of $C^{c}$ having multiplicity $m+1$. This last statement follows easily identifying the linear factors of the homogeneous polynomial $C^{c}(x, y)$ in two variables with the roots of the polynomial $C^{c}(1, z)$ in the variable $z$. Hence, $A^{a}$ is a repeated factor of $C^{c}$. It is in contradiction with the assumption.

We first consider the case that system (1) has a given invariant algebraic curve.

Lemma 6. Assume that polynomial system (1) of degree $m$ has an invariant algebraic curve $C=0$ of degree $c$, and that $C$ satisfies condition $(i)$ of theorem 1. 
(a) If $\left(C_{x}, C_{y}\right)=1$, then system (1) has the following normal form:

$$
\dot{x}=A C-D C_{y} \quad \dot{y}=B C+D C_{x}
$$

where $A, B$ and $D$ are suitable polynomials.

(b) If $C$ satisfies condition (ii) of theorem 1, then system (1) has the normal form (9) with $a, b \leqslant m-c$ and $d \leqslant m-c+1$. Moreover, if the highest order term $C^{c}$ of $C$ does not have the factors $x$ and $y$, then $a \leqslant p-c, b \leqslant q-c$ and $d \leqslant \min \{p, q\}-c+1$.

Proof. (a) Since there are no points at which $C, C_{x}$ and $C_{y}$ vanish simultaneously, from Hilbert's nullstellensatz we obtain that there exist polynomials $E, F$ and $G$ such that

$$
E C_{x}+F C_{y}+G C=1 .
$$

As $C$ satisfies equation (2), we get from (2) and (10) that

$$
K=(K E+G P) C_{x}+(K F+G Q) C_{y} .
$$

Substituting $K$ into (2), we get

$$
[P-(K E+G P) C] C_{x}=-[Q-(K F+G Q) C] C_{y} .
$$

Since $\left(C_{x}, C_{y}\right)=1$, there exists a polynomial $D$ such that

$$
P-(K E+G P) C=-D C_{y} \quad Q-(K F+G Q) C=D C_{x} .
$$

This proves that system (1) has the form (9) with $A=K E+G P$ and $Q=K F+G Q$.

(b) From $(a)$ and lemma 5 we get that system (1) has the normal form (9). Without loss of generality we can assume that $p \leqslant q$.

We first consider the case that $C^{c}$ has neither factor $x$ nor $y$. So we have $\left(C^{c},\left(C^{c}\right)_{x}\right)=1$ and $\left(C^{c},\left(C^{c}\right)_{y}\right)=1$, where $\left(C^{c}\right)_{x}$ denotes the derivative of $C^{c}$ with respect to $x$. In (9) we assume that $a>p-c$, otherwise the statement follows. Then $d=a+1$. Moreover, from the highest order terms of (9) we get

$$
A^{a} C^{c}=D^{a+1} C_{y}^{c-1}
$$

where $C_{y}^{c-1}$ denotes the homogeneous part with degree $c-1$ of $C_{y}$. Since $\left(C^{c}, C_{y}^{c-1}\right)=1$, there exists a polynomial $F$ such that

$$
A^{a}=F C_{y}^{c-1} \quad D^{a+1}=F C^{c} .
$$

In (9) we replace $A$ by $A-F C_{y}$ and $D$ by $D-F C$, so the degrees of polynomials under consideration reduce by one. We continue this process and do the same for $\dot{y}$ until we reach a system of the form

$$
\dot{x}=A C-D C_{y} \quad \dot{y}=B C+E C_{x}
$$

with $a \leqslant p-c, d \leqslant p-c+1, b \leqslant q-c$ and $e \leqslant q-c+1$. Since $C=0$ is an invariant algebraic curve of (11), from (2) we get

$$
C\left(A C_{x}+B C_{y}\right)+C_{x} C_{y}(E-D)=K C .
$$

This implies that there exists a polynomial $R$ such that $E-D=R C$, because $C$ with $C_{x}$ and $C_{y}$ are coprime.

If $e \geqslant d$, then $r=e-c$. We write $B C+E C_{x}=\left(B+R C_{x}\right) C+D C_{x}$ and denote $B+R C_{x}$ again by $B$, then system (11) has the form (9) where $A, B$ and $D$ have the required degrees.

If $e<d$, then $r=d-c$. We write $A C-D C_{y}=\left(A+R C_{y}\right) C-E C_{y}$ and denote $A+R C_{y}$ again by $A$, then system (11) has the form (9) where $A, B$ and $E$ instead of $D$ have the required degrees. This proves the second part of $(b)$. 
Now we prove the first part of $(b)$. We note that even though $C^{c}$ has no repeated factor, $C^{c}$ with $C_{x}^{c-1}$ or $C_{y}^{c-1}$ may have a common factor in $x$ or $y$ (for example, $C^{3}=x\left(x^{2}+y^{2}\right)$, $C^{3}=y\left(x^{2}+y^{2}\right)$ or $\left.C^{4}=x y\left(x^{2}+y^{2}\right)\right)$. In order to avoid this difficulty we rotate system (1) slightly such that $C^{c}$ has no factors in $x$ and $y$. Then, applying the above method to the new system we get that the new system has a normal form (9) with the degrees of $A, B$ and $D$ as those of the second part of $(b)$.

We claim that under affine changes system (9) preserves its form and the upper bound of the polynomials, i.e. $a, b \leqslant m-c$ and $d \leqslant m-c+1$. Indeed, using the affine change of variables $u=a_{1} x+b_{1} y+c_{1}$ and $v=a_{2} x+b_{2} y+c_{2}$ with $a_{1} b_{2}-a_{2} b_{1} \neq 0$, system (9) becomes $\dot{u}=\left(a_{1} A+b_{1} B\right) C-\left(a_{1} b_{2}-a_{2} b_{1}\right) D C_{v} \quad \dot{v}=\left(a_{2} A+b_{2} B\right) C+\left(a_{1} b_{2}-a_{2} b_{1}\right) D C_{u}$.

Hence, the claim follows. This completes the proof of $(b)$, and consequently we have the proof of the lemma.

Lemma 7. Assume that $C=0$ and $D=0$ are different irreducible invariant algebraic curves of system (1) of degree $m$, and that they satisfy conditions ( $i$ ) and (iii) of theorem 1.

(a) If $\left(C_{x}, C_{y}\right)=1$ and $\left(D_{x}, D_{y}\right)=1$, then system (1) has the normal form

$$
\dot{x}=A C D-E C_{y} D-F C D_{y} \quad \dot{y}=B C D+E C_{x} D+F C D_{x}
$$

(b) If $C$ and $D$ satisfy conditions (ii) and (v), then system (1) has the normal form (12) with $a, b \leqslant m-c-d$ and $e, f \leqslant m-c-d+1$.

Proof. Since $(C, D)=1$, the curves $C$ and $D$ have finitely many intersection points. By assumption (i) at each of such points there is at least one nonzero first derivative of both $C$ and $D$. In a similar way to the proof of the claim inside the proof of lemma 6 , we can prove that under an affine change of the variables, system (12) preserves its form and the bound for the degrees of $A, B, E$ and $F$. So, we rotate system (1) slightly such that all first derivatives of $C$ and $D$ are not equal to zero at the intersection points.

From Hilbert's nullstellensatz, there exist polynomials $M_{i}, N_{i}$ and $R_{i}, i=1,2$ such that

$$
M_{1} C+N_{1} D+R_{1} D_{y}=1 \quad M_{2} C+N_{2} D+R_{2} C_{y}=1 .
$$

By lemma 6 we get that

$$
P=A_{1} C-E_{1} C_{y}=G_{1} D-F_{1} D_{y}
$$

for some polynomials $A_{1}, E_{1}, G_{1}$ and $F_{1}$. Moreover, using the first equation of (13) we have $F_{1}=S C+T D+U C_{y}$ for some polynomials $S, T$ and $U$. Substituting $F_{1}$ into (14) we obtain that

$$
\left(A_{1}+S D_{y}\right) C+\left(-G_{1}+T D_{y}\right) D+\left(-E_{1}+U D_{y}\right) C_{y}=0
$$

Using the second equation of (13) and (15) to eliminate $C_{y}$ we get

$$
-E_{1}+U D_{y}=V C+W D
$$

for some polynomials $V$ and $W$. Substituting (16) into (15), we have

$$
\left(A_{1}+S D_{y}+V C_{y}\right) C=\left(G_{1}-T D_{y}-W C_{y}\right) D .
$$

Since $(C, D)=1$, there exists a polynomial $K$ such that

$$
A_{1}+S D_{y}+V C_{y}=K D \quad G_{1}-T D_{y}-W C_{y}=K C .
$$

Substituting $E_{1}$ of (16) and $A_{1}$ of (17) into (14), then we have

$$
P=K C D-S C D_{y}+W C_{y} D-U C_{y} D_{y} .
$$


Similarly, we can prove that there exist some polynomials $K^{\prime}, S^{\prime}, W^{\prime}$ and $U^{\prime}$ such that

$$
Q=K^{\prime} C D+S^{\prime} C D_{x}-W^{\prime} C_{x} D+U^{\prime} C_{x} D_{x} .
$$

Since $C$ is an invariant algebraic curve of (1), we have $P C_{x}+Q C_{y}=K_{C} C$ for some polynomial $K_{C}$. Using (18) and (19) we get

$K_{C} C=C\left[D\left(K C_{x}+K^{\prime} C_{y}\right)-S C_{x} D_{y}+S^{\prime} C_{y} D_{x}\right]+C_{x} C_{y}\left[D\left(W-W^{\prime}\right)-U D_{y}+U^{\prime} D_{x}\right]$.

As $C, C_{x}$ and $C_{y}$ are coprime, there exists a polynomial $Z$ such that

$$
D\left(W-W^{\prime}\right)-U D_{y}+U^{\prime} D_{x}=Z C .
$$

Substituting the expression $D W-U D_{y}$ into (18), we get

$$
P=K C D-S C D_{y}+W^{\prime} C_{y} D-U^{\prime} C_{y} D_{x}+Z C C_{y} .
$$

Since $D=0$ is an invariant algebraic curve of system (1), we have $P D_{x}+Q D_{y}=K_{D} D$ for some polynomial $K_{D}$. Using (19) and (21) we get

$$
\begin{gathered}
K_{D} D=D\left[C\left(K D_{x}+K^{\prime} D_{y}\right)+W^{\prime}\left(C_{y} D_{x}-C_{x} D_{y}\right)\right]+D_{x}\left[C D_{y}\left(-S+S^{\prime}\right)\right. \\
\left.+U^{\prime}\left(C_{x} D_{y}-C_{y} D_{x}\right)+Z C C_{y}\right] .
\end{gathered}
$$

As $D$ and $D_{x}$ are coprime, there exists a polynomial $M$ such that

$$
C D_{y}\left(-S+S^{\prime}\right)+U^{\prime}\left(C_{x} D_{y}-C_{y} D_{x}\right)+Z C C_{y}=M D .
$$

The curves $C$ and $D$ being transversal implies that $C, D$ and $C_{x} D_{y}-C_{y} D_{x}$ have no common zeros. From Hilbert's nullstellensatz, there exist some polynomials $M_{3}, N_{3}$ and $R_{3}$ such that

$$
M_{3} C+N_{3} D+R_{3}\left(C_{x} D_{y}-C_{y} D_{x}\right)=1 .
$$

Eliminating the term $C_{x} D_{y}-C_{y} D_{x}$ from (22) and (23), we obtain that $U^{\prime}=I C+J D$ for some polynomials $I$ and $J$. Hence, equation (22) becomes

$C\left[I\left(C_{x} D_{y}-C_{y} D_{x}\right)+D_{y}\left(-S+S^{\prime}\right)+Z C_{y}\right]+D\left[J\left(C_{x} D_{y}-C_{y} D_{x}\right)-M\right]=0$.

Since $(C, D)=1$, there exists a polynomial $G$ such that

$$
\begin{aligned}
& M=J\left(C_{x} D_{y}-C_{y} D_{x}\right)+G C \\
& I\left(C_{x} D_{y}-C_{y} D_{x}\right)+D_{y}\left(-S+S^{\prime}\right)+Z C_{y}=G D .
\end{aligned}
$$

Substituting $Z C_{y}-S D_{y}$ and $U^{\prime}$ into (21) we obtain that

$$
P=(K+G) C D-\left(I C_{x}+S^{\prime}\right) C D_{y}+\left(W^{\prime}-J D_{x}\right) D C_{y} .
$$

This means that $P$ can be expressed in the form (18) with $U=0$.

Working in a similar way, we can express $Q$ in the form (19) with $U^{\prime}=0$. Thus, (20) is reduced to $D\left(W-W^{\prime}\right)=Z C$. Hence, we have $W=W^{\prime}+H C$ for some polynomial $H$. Consequently, $Z=H D$. Therefore, from (22) we obtain that $C D_{y}\left(-S+S^{\prime}\right)=D\left(M-H C C_{y}\right)$. Since $(C, D)=1$ and $\left(D, D_{y}\right)=1$, we have $S=S^{\prime}+L D$ for some polynomial $L$. Substituting $W$ and $S$ into (18) we obtain that $P$ and $Q$ have the form (12). This proves statement (a).

As in the proof of lemma 6 we can prove that under suitable affine change of variables the form of system (12) and the bound of the degrees of the polynomials $A, B, E$ and $F$ are invariant. So, without loss of generality we can assume that the highest order terms of $C$ and $D$ are neither divisible by $x$ nor $y$.

By the assumptions, the conditions of statement (a) hold, so we get that system (1) has the form (12). If the bounds of the degrees of $A, B, E$ and $F$ are not satisfied, we have by (12) that

$$
\begin{aligned}
& A^{a} C^{c} D^{d}-E^{e} C_{y}^{c-1} D^{d}-F^{f} C^{c} D_{y}^{d-1}=0 \\
& B^{b} C^{c} D^{d}+E^{e} C_{x}^{c-1} D^{d}+F^{f} C^{c} D_{x}^{d-1}=0
\end{aligned}
$$


We remark that if one of the numbers $a+c+d, e+c-1+d$ and $f+c+d-1$ is less than the other two, then its corresponding term in the first equation of (24) is equal to zero. The same remark is applied to the second equation of (24). From the hypotheses it follows that $C^{c}$ and $C_{y}^{c-1}$ are coprime, and also $D^{d}$ and $D_{y}^{d-1}$, and $C^{c}$ and $D^{d}$, respectively. Hence, from these last two equations we obtain that there exist polynomials $K$ and $L$ such that $E^{e}=K C^{c}$, $F^{f}=L D^{d}$ and

$$
A^{a}=K C_{y}^{c-1}+L D_{y}^{d-1} \quad B^{b}=-K C_{x}^{c-1}-L D_{x}^{d-1} .
$$

We rewrite equation (12) as

$$
\begin{aligned}
& \dot{x}=\left(A-K C_{y}-L D_{y}\right) C D-(E-K C) C_{y} D-(F-L D) C D_{y} \\
& \dot{y}=\left(B+K C_{x}+L D_{x}\right) C D+(E-K C) C_{x} D+(F-L D) C D_{x} .
\end{aligned}
$$

Thus, we reduce the degrees of $A, B, E$ and $F$ in (12) by one. We can continue this process until the bounds are reached. This completes the proof of statement $(b)$.

Lemma 8. Let $C_{i}=0$ for $i=1, \ldots$, pe different irreducible invariant algebraic curves of system (1) with deg $C_{i}=c_{i}$. Assume that $C_{i}$ satisfy conditions (i), (iii) and (iv) of theorem 1. Then

(a) If $\left(C_{i x}, C_{i y}\right)=1$ for $i=1, \ldots, p$, then system (1) has the normal form

$$
\dot{x}=\left(B-\sum_{i=1}^{p} \frac{A_{i} C_{i y}}{C_{i}}\right) \prod_{i=1}^{p} C_{i} \quad \dot{y}=\left(D+\sum_{i=1}^{p} \frac{A_{i} C_{i x}}{C_{i}}\right) \prod_{i=1}^{p} C_{i}
$$

where $B, D$ and $A_{i}$ are suitable polynomials.

(b) If $C_{i}$ satisfy conditions (ii) and ( $v$ ) of theorem 1, then system (1) has the normal form (25) with $b, d \leqslant m-\sum_{i=1}^{p} c_{i}$ and $a_{i} \leqslant m-\sum_{i=1}^{p} c_{i}+1$.

Proof. We use induction to prove this lemma. By lemmas 6 and 7 we assume that for any $l$ with $2 \leqslant l<p$ we have

$$
P=\sum_{i=1}^{l}\left(B_{i}-\frac{A_{i} C_{i y}}{C_{i}}\right) \prod_{i=1}^{l} C_{i} \quad Q=\sum_{i=1}^{l}\left(D_{i}+\frac{A_{i} C_{i x}}{C_{i}}\right) \prod_{i=1}^{l} C_{i}
$$

where $\sum_{i=1}^{l} B_{i}=B$ and $\sum_{i=1}^{l} D_{i}=D$. Since $C_{l+1}=0$ is an invariant algebraic curve, from lemma 6 we get that there exist some polynomials $E, G$ and $H$ such that

$$
\begin{aligned}
& P=\sum_{i=1}^{l}\left(B_{i}-\frac{A_{i} C_{i y}}{C_{i}}\right) \prod_{i=1}^{l} C_{i}=E C_{l+1}-G C_{l+1, y} \\
& Q=\sum_{i=1}^{l}\left(D_{i}+\frac{A_{i} C_{i x}}{C_{i}}\right) \prod_{i=1}^{l} C_{i}=H C_{l+1}+G C_{l+1, x} .
\end{aligned}
$$

Now we consider the curves

$$
K_{j}=\prod_{\substack{i=1 \\ i \neq j}}^{l} C_{i}=0 \quad j=1, \ldots, l .
$$

From the assumptions we obtain that there are no points at which all the curves $K_{i}=0$ and $C_{l+1}=0$ intersect. Otherwise, at least three of the curves $C_{i}=0$ for $i=1, \ldots, l+1$ intersect at some point. Hence, there exist polynomials $U$ and $V_{i}$ for $i=1, \ldots, l$ such that

$$
U C_{l+1}+\sum_{i=1}^{l} V_{i} K_{i}=1
$$


Using this equality, we can rearrange (26) as

$$
\begin{aligned}
& \left(E-G U C_{l+1, y}\right) C_{l+1}=\sum_{i=1}^{l}\left(B_{i} C_{i}-A_{i} C_{i y}+G V_{i} C_{l+1, y}\right) K_{i} \\
& \left(H+G U C_{l+1, x}\right) C_{l+1}=\sum_{i=1}^{l}\left(D_{i} C_{i}+A_{i} C_{i x}-G V_{i} C_{l+1, x}\right) K_{i} .
\end{aligned}
$$

Using (27) and (28) to eliminate $C_{l+1}$ we obtain that

$$
E-G U C_{l+1, y}=\sum_{i=1}^{l} I_{i} K_{i} \quad H+G U C_{l+1, x}=\sum_{i=1}^{l} J_{i} K_{i}
$$

for some polynomials $I_{i}$ and $J_{i}$. Substituting these last equalities into (28), we have

$$
\begin{aligned}
& \sum_{i=1}^{l}\left(B_{i} C_{i}-A_{i} C_{i y}+G V_{i} C_{l+1, y}-I_{i} C_{l+1}\right) K_{i}=0 \\
& \sum_{i=1}^{l}\left(D_{i} C_{i}+A_{i} C_{i x}-G V_{i} C_{l+1, x}-J_{i} C_{l+1}\right) K_{i}=0 .
\end{aligned}
$$

It is easy to check that the expressions multiplying $K_{i}$ in the two summations of (29) are divisible by $C_{i}$. Hence, there exist polynomials $L_{i}$ and $F_{i}$ for $i=1, \ldots, l$ such that

$$
\begin{aligned}
& B_{i} C_{i}-A_{i} C_{i y}+G V_{i} C_{l+1, y}-I_{i} C_{l+1}=L_{i} C_{i} \\
& D_{i} C_{i}+A_{i} C_{i x}-G V_{i} C_{l+1, x}-J_{i} C_{l+1}=F_{i} C_{i} .
\end{aligned}
$$

So, from (29) we get that $\sum_{i=1}^{l} L_{i}=0$ and $\sum_{i=1}^{l} F_{i}=0$. This implies that (26) can be rewritten as

$P=\sum_{i=1}^{l}\left(\left(B_{i}-L_{i}\right) C_{i}-A_{i} C_{i y}\right) K_{i} \quad Q=\sum_{i=1}^{l}\left(\left(C_{i}-F_{i}\right) C_{i}+A_{i} C_{i x}\right) K_{i}$.

Moreover, we write (30) in the form

$$
\begin{aligned}
& \left(B_{i}-L_{i}\right) C_{i}-A_{i} C_{i y}=I_{i} C_{l+1}-G V_{i} C_{l+1, y}=P_{i} \\
& \left(D_{i}-F_{i}\right) C_{i}+A_{i} C_{i x}=J_{i} C_{l+1}+G V_{i} C_{l+1, x}=Q_{i} .
\end{aligned}
$$

It is easy to see that $C_{i}$ and $C_{l+1}$ are invariant algebraic curves of the system $\dot{x}=P_{i}, \dot{y}=$ $Q_{i}$. So, from statement (a) of lemma 7 we can obtain that

$$
\begin{aligned}
P_{i} & =\left(B_{i}-L_{i}\right) C_{i}-A_{i} C_{i y}=X_{i} C_{i} C_{l+1}-Y_{i} C_{i y} C_{l+1}-N_{i} C_{i} C_{l+1, y} \\
Q_{i} & =\left(D_{i}-F_{i}\right) C_{i}+A_{i} C_{i x}=Z_{i} C_{i} C_{l+1}+Y_{i} C_{i x} C_{l+1}+N_{i} C_{i} C_{l+1, x} .
\end{aligned}
$$

Substituting these last two equations into (31), we obtain that system (1) has the form (25) with the $l+1$ invariant algebraic curves $C_{1}, \ldots, C_{l+1}$. From induction we have finished the proof of statement (a).

The proof of statement (b) is almost identical with those of lemma 7(b), so we shall omit it here. Hence, this ends the proof of the lemma.

Proof of theorem. From lemma 8 it follows statement (a) of theorem 1.

By checking the degrees of polynomials $A_{i}, B$ and $D$ in statement (b) of lemma 8 we obtain statement (b) of theorem 1.

From statement (a) of lemma 8 , we can rearrange system (1) such that it has the form (25). But from statement (b) of lemma 8 we must have $B=0, D=0$ and $A_{i}=0$. This proves statement (c) of theorem 1. 


\section{Proof of theorem 2.}

The proof is formed by the following examples. First, we consider the case $r<m+1$. That is, the sum of degrees of the given invariant algebraic curves is less than the degree of the system plus one.

Example 1. The algebraic curve $C=y^{3}+x^{3}-x^{2}=0$ satisfies all conditions of theorem 1 excepting (i). The cubic system

$$
\dot{x}=2 x-2 x^{3}-3 x y^{2}+y^{3} \quad \dot{y}=\frac{4}{3} y+x^{2}-3 x^{2} y-3 y^{3}
$$

has $C$ as an invariant algebraic curve. We claim that system (33) does not have the form (5). Otherwise, it can be written in the form

$$
\begin{aligned}
& \dot{x}=A\left(y^{3}+x^{3}-x^{2}\right)+D\left(-3 y^{2}\right) \\
& \dot{y}=B\left(y^{3}+x^{3}-x^{2}\right)+D\left(3 x^{2}-2 x\right)
\end{aligned}
$$

where $A, B$ and $D$ are polynomials. It is in contradiction with (33), because in the first equation of (33) there is a linear term.

Example 2. The algebraic curve $C=y-x^{2}=0$ satisfies all conditions of theorem 1 excepting (ii). The polynomial system of degree $m$ with $m \geqslant 2$ :

$\dot{x}=D(y)+x E(y)+A(x, y)\left(y-x^{2}\right) \quad \dot{y}=2 x D(y)+2 y E(y)+B(x, y)\left(y-x^{2}\right)$

has $C$ as an invariant algebraic curve, where $\operatorname{deg} D, \operatorname{deg} E=m-1, \operatorname{and} \operatorname{deg} A, \operatorname{deg} B \leqslant m-2$. We can write system (34) in the form (5), i.e.

$$
\begin{aligned}
& \dot{x}=A(x, y)\left(y-x^{2}\right)+D(y)+x E(y) \\
& \dot{y}=(B(x, y)+2 E(y))\left(y-x^{2}\right)+2 x(D(y)+x E(y)) .
\end{aligned}
$$

But then $\operatorname{deg}(B(x, y)+2 E(y))=m-1>m-\operatorname{deg} C$.

Example 3. The algebraic curves $C_{1}=x^{2}+y^{2}-1=0$ and $C_{2}=y-1=0$ satisfy all conditions of theorem 1 excepting (iii). The cubic system

$\dot{x}=-1-y+x^{2}+x y+y^{2}+x^{2} y+y^{3}=P \quad \dot{y}=\left(y+x^{2}+y^{2}\right)(y-1)=Q$

has $C_{1}$ and $C_{2}$ as invariant algebraic curves. We claim that system (35) cannot be written in the form (5). Otherwise, $Q$ can be written as

$$
Q=B\left(x^{2}+y^{2}-1\right)(y-1)+D 2 x(y-1)
$$

where $B$ and $D$ are polynomials. However, there do not exist polynomials $B$ and $D$ such that

$$
B\left(x^{2}+y^{2}-1\right)+2 x D=y+x^{2}+y^{2} .
$$

Because if the equality holds, then $B$ must contain the monomial $-y$. Let $a y^{t}$ be the monomial of $B$ with the highest degree $t \geqslant 1$ and without the variable $x$. Then the left-hand side of (36) contains the monomial $a y^{t+2}$. It is in contradiction with the right-hand side of (36).

Example 4. The algebraic curves $C_{1}=x=0, C_{2}=y=0$ and $C_{3}=x+y=0$ satisfy all conditions of theorem 1 excepting (iv). The cubic system

$$
\dot{x}=\left(1+x+y+x^{2}+x y\right) x=P \quad \dot{y}=\left(1+x^{2}+2 x y+y^{2}\right) y=Q
$$


has these three curves as invariant algebraic curves. We claim that system (37) cannot be written in the form (5). Otherwise, the polynomial $Q$ can be written as

$$
Q=B x y(x+y)+D y(x+y)+E x y
$$

where $B, D$ and $E$ are polynomials. But it is in contradiction with (37).

Example 5. The algebraic curves $C_{1}=x y+1=0$ and $C_{2}=y=0$ satisfy all conditions of theorem 1 excepting $(\mathrm{v})$. The cubic system

$\dot{x}=1+x+y-x^{2}+x^{3}+2 x y^{2}=P \quad \dot{y}=\left(x+y-x^{2}+x y-y^{2}\right) y=Q$

has $C_{1}$ and $C_{2}$ as invariant algebraic curves. If we write this system in the form (5), then we have

$$
Q=B(x y+1) y+D y^{2}
$$

where $B$ and $C$ are polynomials. Comparing it with (38), we get that $B$ cannot be a constant. So, $\operatorname{deg} B>0=m-r$, which is in contradiction with statement (a) of theorem 1 .

Next, we consider the case $r=m+1$. That is, the sum of the degrees of the given invariant algebraic curves is equal to the degree of the system plus one.

Example 6. The curve $C=x^{2}+x^{3}+y^{3}=0$ satisfies all conditions of theorem 1 excepting (i). The quadratic systems with $C$ as an invariant algebraic curve can be written as

$$
\dot{x}=\frac{3}{2} a x+\frac{3}{2} a x^{2}-b y^{2} \quad \dot{y}=\frac{2}{3} b x+a y+b x^{2}+\frac{3}{2} a x y
$$

where $a$ and $b$ are arbitrary complex numbers. Obviously, if $a \neq 0$ this system cannot have the form (6).

Example 7. The curve $C=y-x^{3}=0$ satisfies all conditions of theorem 1 excepting (ii). It is an invariant algebraic curve of the system

$$
\dot{x}=1+x-x^{2}+x y \quad \dot{y}=3 y+3 x^{2}-3 x y+3 y^{2} .
$$

This system cannot be written in the form (6).

Example 8. The curves $C_{1}=x^{2}+y^{2}-1=0$ and $C_{2}=y-1=0$ satisfy all conditions of theorem 1 excepting (iii). Moreover, $C_{1}$ and $C_{2}$ are invariant algebraic curves of system (7). However, system (7) does not have the form (6) if $a \neq 0$.

Example 9. The curves $C_{1}=x+\mathrm{i} y=0, C_{2}=x-\mathrm{i} y=0$ and $C_{3}=x=0$ satisfy all conditions of theorem 1 excepting (iv). The quadratic system

$$
\dot{x}=-b\left(x^{2}+y^{2}\right)+x+y(a x+b y) \quad \dot{y}=k\left(x^{2}+y^{2}\right)+y-x(a x+b y)
$$

has $C_{1}, C_{2}$ and $C_{3}$ as invariant algebraic curves, but this system cannot take the form (6).

Example 10. The curves $C_{1}=x y-1=0$ and $C_{2}=x=0$ satisfy all conditions of theorem 1 excepting (v). They are invariant algebraic curves of the system

$$
\dot{x}=(1-2 x+y) x \quad \dot{y}=1-y+x y-y^{2} .
$$

Obviously, this system does not have the form (6).

Last we give the counterexamples for the case $r>m+1$. That is, the sum of the degrees of the invariant algebraic curves is larger than the degree of the system plus one.

Example 11. The algebraic curve $C=x^{4}+x^{3}+y^{4}=0$ satisfies all conditions of theorem 1 excepting (i). The quadratic systems having $C$ as an invariant algebraic curve are

$$
\dot{x}=a x+a x^{2} \quad \dot{y}=\frac{3}{4} a y+a x y .
$$

So, statement (c) of theorem 1 is not satisfied. 
Example 12. The algebraic curve $C=y-x^{4}=0$ satisfies all conditions of theorem 1 excepting (ii). The quadratic systems having $C$ as an invariant algebraic curve are

$$
\dot{x}=a x+b x^{2}+c x y \quad \dot{y}=4 a y+4 b x y+4 c y^{2} .
$$

They are not zero unless $a=b=c=0$.

Example 13. The algebraic curves $C_{1}=x^{2}+y^{2}-1=0, C_{2}=y-1=0$ and $C_{3}=4 x+3 y+5=0$ satisfy all conditions of theorem 1 excepting (iii). However, the quadratic system $\dot{x}=y(2 x-y+1)+x^{2}+y^{2}-1, \dot{y}=x(y-1)+2 y^{2}-2$ has these three curves as invariant algebraic curves.

Example 14. The algebraic curves $C_{1}=x=0, C_{2}=y=0$ and $C_{3}=x+y=0$ satisfy all conditions of theorem 1 excepting (iv). The linear systems having these three curves as invariant algebraic curves are $\dot{x}=a x, \dot{y}=a y$. They are not zero unless $a=0$.

Example 15. The algebraic curves $C_{1}=x y-1, C_{2}=y$ and $C_{3}=y+1$ satisfy all conditions of theorem 1 excepting (v). The quadratic system with $C_{1}, C_{2}$ and $C_{3}$ as invariant algebraic curves are

$$
\dot{x}=a-b x-(a+b) x y \quad \dot{y}=b y(y+1) .
$$

They are not zero unless $a=b=0$.

From these 15 examples follows the proof of theorem 2.

\section{Proof of theorem 3.}

The proof is separated into three parts. The first part shows that there exists a set $\Omega_{1}$ of values of the parameters $a$ and $b$ such that system (7) has only the given two invariant algebraic curves. The second part give a proof that there exists a set $\Omega_{2}$ of values of $a$ and $b$ such that systems (7) have no exponential factors. Moreover, $\Omega_{1} \cap \Omega_{2} \neq \varnothing$. The last step contributes to prove that system (7) is not Darboux integrable for $a, b \in \Omega_{1} \cap \Omega_{2}$.

We need the following result (for a proof, see [10]).

Lemma 9. Assume that system (1) with degree $m$ has an invariant algebraic curve $C$ of degree $n$. Let $C_{n}, P_{m}$ and $Q_{m}$ be the homogeneous parts of $C$ with degree $n, P$ and $Q$ with degree $m$. Then the irreducible factor of $C_{n}$ divides y $P_{m}-x Q_{m}$.

The first part is formed by the following proposition, which is related to the existence of invariant algebraic curves of system (7).

Proposition 10. For each $b \neq 1 \pm \frac{1}{p}$ with $p \in \mathbb{N}$ there exists a numerable set $\Upsilon$ such that if $a \in \mathbb{R} \backslash(\Upsilon \cup\{0\})$, then system (7) has no irreducible invariant algebraic curves different from $f_{1}=0$ and $f_{2}=0$.

Proof. Assume that $C=\sum_{i=0}^{n} C_{i}(x, y)=0$ is an invariant algebraic curve of system (7) with cofactor $K=K_{1}+K_{0}$, where $C_{i}$ and $K_{i}$ are homogeneous polynomials of degree $i$. From the definition of the invariant algebraic curve, i.e. (2), we have

$$
\left[x^{2}+a x y+(1-b) y^{2}+b y-1\right] \sum_{i=1}^{n} C_{i x}+\left[b x y+a y^{2}-b x-a\right] \sum_{i=1}^{n} C_{i y}=\left(K_{1}+K_{0}\right) \sum_{i=0}^{n} C_{i} .
$$


Equating the terms with the same degree we obtain

$$
\begin{gathered}
L\left[C_{n-i}\right]=K_{1} C_{n-i}+K_{0} C_{n-i+1}-b y C_{n-i+1, x}+b x C_{n-i+1, y}+C_{n-i+2, x}+a C_{n-i+2, y} \\
i=0,1, \ldots, n+2
\end{gathered}
$$

where $C_{i}=0$ for $i<0$ and $i>n$, and $L$ is the partial differential operator

$$
L=\left[x^{2}+a x y+(1-b) y^{2}\right] \frac{\partial}{\partial x}+\left[b x y+a y^{2}\right] \frac{\partial}{\partial y} .
$$

For system (7) we have $y P_{2}-x Q_{2}=(1-b) y\left(x^{2}+y^{2}\right)$. So, from lemma 9 we can assume that

$$
C_{n}=\left(x^{2}+y^{2}\right)^{l} y^{m} \quad n=2 l+m .
$$

Substituting $C_{n}$ into (39) with $i=0$ and doing some computations we get

$$
K_{1}=(2 l+m b) x+a(2 l+m) y .
$$

Set $C_{n-1}=\sum_{i=0}^{n-1} c_{n-1-i} x^{n-1-i} y^{i}$. Substituting $C_{n-1}, C_{n}$ and $K_{1}$ into (39) with $i=1$ and doing some calculations, we obtain

$$
\begin{aligned}
\sum_{i=0}^{n-1}(m-1- & i+i b-m b) c_{n-1-i} x^{2 l+m-i} y^{i}-\sum_{i=0}^{n-1} a c_{n-1-i} x^{2 l+m-1-i} y^{i+1} \\
& +\sum_{i=0}^{n-1}(2 l+m-1-i)(1-b) c_{n-1-i} x^{2 l+m-2-i} y^{i+2} \\
= & K_{0}\left(x^{2}+y^{2}\right)^{l} y^{m}+m b x\left(x^{2}+y^{2}\right)^{l} y^{m-1} \\
= & \sum_{i=0}^{l} K_{0}\left(\begin{array}{l}
l \\
i
\end{array}\right) x^{2 l-2 i} y^{m+2 i}+\sum_{i=0}^{l} m b\left(\begin{array}{l}
l \\
i
\end{array}\right) x^{2 l+1-2 i} y^{m+2 i-1}
\end{aligned}
$$

This equation can be written as

$$
\begin{gathered}
\sum_{i=0}^{n}\left[(m-1-i+i b-m b) c_{n-1-i}-a c_{n-i}+(2 l+m+1-i)(1-b) c_{n+1-i}\right] x^{2 l+m-i} y^{i} \\
=\sum_{i=0}^{l} K_{0}\left(\begin{array}{l}
l \\
i
\end{array}\right) x^{2 l-2 i} y^{m+2 i}+\sum_{i=0}^{l} m b\left(\begin{array}{l}
l \\
i
\end{array}\right) x^{2 l+1-2 i} y^{m+2 i-1}
\end{gathered}
$$

where $c_{i}=0$ for $i<0$ and $i>n-1$. Equating the coefficients of $x^{i} y^{j}$ in the above equation, we get

$$
\begin{gathered}
{[m-i-1+(i-m) b] c_{2 l+m-1-i}-a c_{2 l+m-i}+(2 l+m+1-i)(1-b) c_{2 l+m+1-i}=0} \\
i=0,1, \ldots, m-2 \\
{[(2 i-1) b-2 i] c_{2 l-2 i}-a c_{2 l+1-2 i}-(2 l+2-2 i)(b-1) c_{2 l+2-2 i}=m b\left(\begin{array}{l}
l \\
i
\end{array}\right)} \\
i=0,1, \ldots, l \\
{[2 i(b-1)-1] c_{2 l-2 i-1}-a c_{2 l-2 i}-(2 l+1-2 i)(b-1) c_{2 l+1-2 i}=K_{0}\left(\begin{array}{l}
l \\
i
\end{array}\right)} \\
i=0,1, \ldots, l
\end{gathered}
$$


From the assumptions and (40) we can prove easily that $c_{2 l+j}=0$ for $j=1, \ldots, m-1$. Equations (41) and (42) can be written as

$$
\begin{aligned}
& c_{2 l-2 i}=\frac{1}{2 i(b-1)-b}\left[a c_{2 l+1-2 i}+(2 l+2-2 i)(b-1) c_{2 l+2-2 i}+m b\left(\begin{array}{l}
l \\
i
\end{array}\right)\right] \\
& c_{2 l-2 i-1}=\frac{1}{2 i(b-1)-1}\left[a c_{2 l-2 i}+(2 l+1-2 i)(b-1) c_{2 l+1-2 i}+K_{0}\left(\begin{array}{l}
l \\
i
\end{array}\right)\right]
\end{aligned}
$$

with $i=0,1, \ldots, l$. It is easy to check that

From (43) with $i=1$ we get that

$$
c_{2 l}=-m \quad c_{2 l-1}=a m-K_{0} .
$$

$c_{2 l-2}=\frac{a}{b-2}\left(a m-K_{0}\right)-m l=\mathcal{B}_{1}(a, b, l)\left(a m-K_{0}\right)-m\left(\begin{array}{l}l \\ 1\end{array}\right)$

$c_{2 l-3}=\left[\frac{a^{2}}{(2 b-3)(b-2)}+l-\frac{b-1}{2 b-3}\right]\left(a m-K_{0}\right)=\mathcal{B}_{2}(a, b, l)\left(a m-K_{0}\right)$.

In what follows we use induction to find the coefficients $c_{2 l-i}$ for $i=4, \ldots, 2 l$. Assume that for $i=h$ we have

$c_{2 l-2 h}=\mathcal{B}_{2 h-1}(a, b, l)\left(a m-K_{0}\right)-m\left(\begin{array}{l}l \\ h\end{array}\right) \quad c_{2 l-1-2 h}=\mathcal{B}_{2 h}(a, b, l)\left(a m-K_{0}\right)$

where $\mathcal{B}_{j-1}(a, b, l)$ for $j=2 h, 2 h+1$, are polynomials in $a$ where coefficients are functions of $b$ and $l$ and the highest order terms of the form

$$
a^{j-1} / \prod_{i=2}^{j}[(i-1) b-i]
$$

Then from (43) with $i=h+1$ we get

$$
\begin{aligned}
& c_{2 l-2 h-2}= \frac{1}{2(h+1)(b-1)-b}\left\{a \mathcal{B}_{2 h}(a, b, l)\left(a m-K_{0}\right)+(2 l-2 h)(b-1)\right. \\
&\left.\quad \times\left[\mathcal{B}_{2 h-1}(a, b, l)\left(a m-K_{0}\right)-m\left(\begin{array}{l}
l \\
h
\end{array}\right)\right]+m b\left(\begin{array}{c}
l \\
h+1
\end{array}\right)\right\} \\
&=\mathcal{B}_{2 h+1}(a, b, l)\left(a m-K_{0}\right)-m\left(\begin{array}{c}
l \\
h+1
\end{array}\right) \\
& c_{2 l-2 h-3}=\frac{1}{2(h+1)(b-1)-1}\left\{a\left[\mathcal{B}_{2 h+1}(a, b, l)\left(a m-K_{0}\right)-m\left(\begin{array}{c}
l \\
h+1
\end{array}\right)\right]\right. \\
&\left.\quad+(2 l-2 h-1)(b-1) \mathcal{B}_{2 h}(a, b, l)\left(a m-K_{0}\right)+K_{0}\left(\begin{array}{c}
l \\
h+1
\end{array}\right)\right\} \\
&= \mathcal{B}_{2 h+2}(a, b, l)\left(a m-K_{0}\right) .
\end{aligned}
$$

where

$$
\begin{aligned}
\mathcal{B}_{2 h+1} & =\frac{1}{2(h+1)(b-1)-b}\left[a \mathcal{B}_{2 h}+(2 l-2 h)(b-1) \mathcal{B}_{2 h-1}\right] \\
\mathcal{B}_{2 h+2} & =\frac{1}{2(h+1)(b-1)-1}\left[a \mathcal{B}_{2 h+1}-\left(\begin{array}{c}
l \\
h+1
\end{array}\right)+(2 l-2 h-1)(b-1) \mathcal{B}_{2 h}\right]
\end{aligned}
$$

are polynomials in $a$ of degree $2 h+1$ and $2 h+2$ respectively, in which the highest order terms are the form (44) for $j=2 h+2$ and $j=2 h+3$, respectively. Hence, from (43) and using induction we obtain that for $h=0,1, \ldots, 2 l$

$$
c_{2 l-h}=\mathcal{B}_{h-1}(a, b, l)\left(a m-K_{0}\right)+\frac{(-1)^{h+1}-1}{2} m\left(\begin{array}{c}
l \\
h / 2
\end{array}\right) .
$$


Moreover, from the first equation of (43) with $i=l$, i.e. $a c_{0}+(b-1) c_{1}+K_{0}=0$ we get

$$
a\left[\mathcal{B}_{2 l-1}\left(a m-K_{0}\right)-m\right]+(b-1) \mathcal{B}_{2 l-2}\left(a m-K_{0}\right)=K_{0} .
$$

This means that

$$
\left[a \mathcal{B}_{2 l-1}+(b-1) \mathcal{B}_{2 l-2}-1\right]\left(a m-K_{0}\right)=0 .
$$

Since $a \mathcal{B}_{2 l-1}+(b-1) \mathcal{B}_{2 l-2}-1$ is a polynomial of degree $2 l$ in the variable $a$, it has at most $2 l$ real roots, denoted by $S_{l}$ the set of the roots. Then, for $a \in \mathbb{R} \backslash S_{l}$ we must have $K_{0}=a m$.

Obviously, $\Upsilon=\cup_{l=1}^{\infty} S_{l}$ is a numerable set. Moreover, for each $a \in \mathbb{R} \backslash \Upsilon$ and $l \in \mathbb{N}$ we have $K_{0}=a m$. So, if $C$ is an invariant algebraic curve of the above form, it has the cofactor $K=K_{1}+K_{0}=(2 l+m b) x+a(2 l+m) y+a m=2(x+a y) l+(b x+a y+a) m$.

Moreover, we can check that $C^{*}=\left(x^{2}+y^{2}-1\right)^{l}(y-1)^{m}=0$ is an invariant algebraic curve with cofactor $K$. If $D=C-C^{*} \neq 0$, then $D=0$ is also an invariant algebraic curve with the cofactor $K$. But $D$ has degree $d \leqslant 2 l+m-2$. Again using lemma 9 we can assume that the highest order homogeneous term of $D$ is of the form $D_{d}=\left(x^{2}+y^{2}\right)^{l^{\prime}} y^{m^{\prime}}$ with $d=2 l^{\prime}+m^{\prime}$. Then, from the above proof we should have the linear part of $K$ is $K_{1}=\left(2 l^{\prime}+m^{\prime} b\right) x+a\left(2 l^{\prime}+m^{\prime}\right) y$. It is in contradiction with the last paragraph. Hence, we must have $C=C^{*}$. This proves that for $b \neq 1 \pm \frac{1}{p}$ with $p \in \mathbb{N}$ and $a \in \mathbb{R} \backslash(\Upsilon \cup\{0\})$ system (7) has only the irreducible invariant algebraic curves $x^{2}+y^{2}=1$ and $y=1$. This proves the proposition.

Now we consider the exponential factors. We recall that if $F=\exp (G / H)$ is an exponential factor of system (1) with cofactor $L$, then $H=0$ is an invariant algebraic curve of system (1) with a cofactor $K_{H}$, and $G$ satisfies the following equation:

$$
P G_{x}+Q G_{y}=K_{H} G+L H .
$$

For more details see [13].

Proposition 11. For each $b \notin \mathbb{Q}$ there exists a numerable set $\Upsilon^{*} \supset \Upsilon$ such that if $a \in \mathbb{R} \backslash\left(\Upsilon^{*} \cup\{0\}\right)$, then system (7) has no exponential factors.

Proof. From proposition 10 system (7) has only the invariant algebraic curves $C_{1}=$ $x^{2}+y^{2}-1=0$ and $C_{2}=y-1=0$. If system (7) has an exponential factor, we can assume that it has the form $F=\exp \left(\frac{G}{C_{1}^{I_{1}} C_{2}^{l_{2}}}\right)$ with a cofactor $L$, where $l_{1}$ and $l_{2}$ are non-negative integers. Since the invariant algebraic curve $C_{1}^{l_{1}} C_{2}^{l_{2}}=0$ has the cofactor $K=l_{1} K_{1}+l_{2} K_{2}=2 l_{1}(x+a y)+l_{2}(b x+a y+a)$, from (45) we get that $G$ satisfies the following equation:

$$
\begin{aligned}
{\left[x^{2}+a x y+\right.} & \left.(1-b) y^{2}+b y-1\right] G_{x}+\left(b x y+a y^{2}-b x-a\right) G_{y} \\
& =\left[2 l_{1}(x+a y)+l_{2}(b x+a y+a)\right] G+L\left(x^{2}+y^{2}-1\right)^{l_{1}}(y-1)^{l_{2}} .
\end{aligned}
$$

Set $\operatorname{deg} G=n$. Since $\operatorname{deg} L \leqslant 1$, we can assume that $L=L_{1}+L_{0}$, where $L_{i}$ are homogeneous polynomials of degree $i$.

Case 1: $n+1<2 l_{1}+l_{2}$. By equating the homogeneous terms of highest degree in (46) we obtain first that $L_{1}=0$, and after that $L_{0}=0$, and so $L=0$. Thus $G$ is an invariant algebraic curve. Moreover, from the assumption of this proposition we obtain that $G=c C_{1}^{l_{1}} C_{2}^{l_{2}}$, where $c$ is a constant. Then, $F=$ constant, and it cannot be an exponential factor.

Case 2: $n+1=2 l_{1}+l_{2}$. Then we have $L_{1}=0$. Set $G=\sum_{i=0}^{n} G_{i}(x, y)$ with $G_{i}$ homogeneous polynomials of degree $i$ and $G_{n}=\sum_{i=0}^{n} a_{i} x^{n-i} y^{i}$, where $a_{i}$ are constants. Then, equating the 
terms of (46) with degree $n+1$ we get that

$$
\begin{gathered}
{\left[x^{2}+a x y+(1-b) y^{2}\right] \sum_{i=0}^{n}(n-i) a_{i} x^{n-i-1} y^{i}+\left(b x y+a y^{2}\right) \sum_{i=0}^{n} i a_{i} x^{n-i} y^{i-1}} \\
=\left[2 l_{1}(x+a y)+l_{2}(b x+a y)\right] \sum_{i=0}^{n} a_{i} x^{n-i} y^{i}+L_{0}\left(x^{2}+y^{2}\right)^{l_{1}} y^{l_{2}} .
\end{gathered}
$$

Using the relation $n+1=2 l_{1}+l_{2}$ we can write this last equation as

$$
\begin{gathered}
\sum_{i=0}^{2 l_{1}+l_{2}+1}\left\{\left[(b-1)\left(i-l_{2}\right)-1\right] a_{i}+a a_{i-1}+(1-b)\left(2 l_{1}+l_{2}+1-i\right) a_{i-2}\right\} x^{2 l_{1}+l_{2}-i} y^{i} \\
=L_{0} \sum_{i=0}^{l_{1}}\left(\begin{array}{c}
l_{1} \\
i
\end{array}\right) x^{2 l_{1}-2 i} y^{2 i+l_{2}}
\end{gathered}
$$

where $a_{i}=0$ for $i<0$ and $i>n$. The last equation is equivalent to

$$
\begin{aligned}
& {\left[(b-1)\left(i-l_{2}\right)-1\right] a_{i}+a a_{i-1}+(1-b)\left(2 l_{1}+l_{2}+1-i\right) a_{i-2}=0} \\
& i=0,1, \ldots, l_{2}-1 \\
& \sum_{j=0}^{2 l_{1}+1}\left\{[(b-1) j-1] a_{j+l_{2}}+a a_{j+l_{2}-1}-(1-b)\left(2 l_{1}+1-j\right) a_{j+l_{2}-2}\right\} x^{2 l_{1}-j} y^{j+l_{2}} \\
& =L_{0} \sum_{i=0}^{l_{1}}\left(\begin{array}{c}
l_{1} \\
i
\end{array}\right) x^{2 l_{1}-2 i} y^{2 i+l_{2}} .
\end{aligned}
$$

Since $b \neq 1 \pm \frac{1}{k}$ for $k \in \mathbb{N}$, from (47) we get that $a_{i}=0$ for $i=0,1, \ldots, l_{2}-1$. From (48) we obtain that for $i=0,1, \ldots, l_{1}$

$[(b-1)(2 i+1)-1] a_{2 i+1+l_{2}}+a a_{2 i+l_{2}}-(1-b)\left(2 l_{1}-2 i\right) a_{2 i+l_{2}-1}=0$

$[(b-1) 2 i-1] a_{2 i+l_{2}}+a a_{2 i+l_{2}-1}-(1-b)\left(2 l_{1}+1-2 i\right) a_{2 i+l_{2}-2}=L_{0}\left(\begin{array}{c}l_{1} \\ i\end{array}\right)$.

Solving (49) for $i=0,1, \ldots, l_{1}-1$ and its second equation with $i=l_{1}$ we get that

$$
a_{l_{2}+h}=\overline{\mathcal{B}}_{h}(a) L_{0} \quad k=0,1, \ldots, 2 l_{1}
$$

where $\overline{\mathcal{B}}_{h}(a)$ is a polynomial of degree $h$ in $a$ whose coefficients are rational functions in $b$ and $l_{1}$. The highest order term of $\overline{\mathcal{B}}_{h}(a)$ in $a$ is $-1 / \prod_{j=0}^{h}[(1-b) j+1]$. So the first equation of (49) with $i=l_{1}$ is $a \overline{\mathcal{B}}_{2 l_{1}} L_{0}=0$. Since the coefficient of $L_{0}$ is a polynomial of degree $m+1$, there exist at most $m+1$ values of $a$ such that it is equal to zero. We denote by $\bar{\gamma}_{l_{1}}$ the set of such $a$. Hence, if $a \notin \bar{\gamma}_{l_{1}}$ we must have $L_{0}=0$. This means that $L=0$. So, system (7) has no exponential factors for $a \notin \bar{\gamma}_{l_{1}}$.

Case 3: $n=2 l_{1}+l_{2}$. Let $L_{1}=L_{10} x+L_{01} y$. Using the notations for $G$ and $G_{n}$ introduced in the study of case 2, equating the terms of (46) with degree $n+1$ and doing some computations we get that

$$
\begin{aligned}
& \left.\sum_{i=0}^{n+2}\left[(b-1)\left(i-l_{2}\right)\right] a_{i}+(1-b)(n+2-i) a_{i-2}\right] x^{n+1-i} y^{i} \\
& =L_{10} \sum_{i=0}^{l_{1}}\left(\begin{array}{c}
l_{1} \\
i
\end{array}\right) x^{2 l_{1}-2 i+1} y^{2 i+l_{2}}+L_{01} \sum_{i=0}^{l_{1}}\left(\begin{array}{c}
l_{1} \\
i
\end{array}\right) x^{2 l_{1}-2 i} y^{2 i+l_{2}+1}
\end{aligned}
$$


where $a_{i}=0$ for $i<0$ and $i>n$. These equations are equivalent to

$$
\begin{aligned}
& \left(i-l_{2}\right) a_{i}-(n+2-i) a_{i-2}=0, \quad i=0,1, \ldots, l_{2}-1 \\
& (b-1) 2 j a_{2 j+l_{2}}+(1-b)\left(2 l_{1}+2-2 j\right) a_{2 j+l_{2}-2}=L_{10}\left(\begin{array}{c}
l_{1} \\
j
\end{array}\right) \\
& (b-1)(2 j+1) a_{2 j+l_{2}+1}+(1-b)\left(2 l_{1}+1-2 j\right) a_{2 j+l_{2}-1}=L_{01}\left(\begin{array}{c}
l_{1} \\
j
\end{array}\right)
\end{aligned}
$$

where $j=0,1, \ldots, l_{1}$.

From the first equation of (50) we obtain that $a_{i}=0$ for $i=0,1, \ldots, l_{2}-1$. Hence, the first equation of (50) with $j=0$ induces to $L_{10}=0$. Thus, we have

$$
a_{2 j+l_{2}}=\frac{2 l_{1}+2-2 j}{2 j} a_{2 j+l_{2}-2} \quad j=1, \ldots, l_{1} .
$$

i.e. $a_{l_{2}+2 j}=\left(\begin{array}{c}l_{1} \\ j\end{array}\right) a_{l_{2}}, j=1, \cdots l_{1}$. From the second equation of (50) with $j=0,1, \ldots, l_{1}-1$ and using induction, we can prove that

$$
a_{2 j+1+l_{2}}=\frac{\mu_{j}}{b-1} L_{01} \quad j=0,1, \ldots, l_{1}-1
$$

with $\mu_{j}>0$. Now the second equation of (50) with $j=l_{1}$ can be written as $\left(1+\mu_{l_{1}}\right) L_{01}=0$. This implies that $L_{01}=0$. Moreover, we have $a_{2 j+1+l_{2}}=0$ for $j=0,1, \ldots, l_{1}-1$.

From the above calculations we get that $L=L_{0}$ and

$$
G_{n}=\sum_{i=0}^{n} a_{i} x^{n-i} y^{i}=a_{l_{2}}\left(x^{2}+y^{2}\right)^{l_{1}} y^{l_{2}} .
$$

Since $a_{l_{2}} \neq 0$, without loss of generality we assume that $a_{l_{2}}=1$.

Equating the terms of (46) with degree $n$ we get that

$$
\begin{gathered}
{\left[x^{2}+a x y+(1-b) y^{2}\right] G_{n-1, x}+\left(b x y+a y^{2}\right) G_{n-1, y}=\left[2 l_{1}(x+a y)+l_{2}(b x+a y)\right] G_{n-1}} \\
-b y G_{n x}+b x G_{n y}+l_{2} a G_{n}+L_{0}\left(x^{2}+y^{2}\right)^{l_{1}} y^{l_{2}} .
\end{gathered}
$$

Let $G_{n-1}=\sum_{i=0}^{n-1} b_{i} x^{n-1-i} y^{i}$. Substituting $G_{n-1}$ into the above equation and doing some computations, we can obtain that

$$
\begin{aligned}
\sum_{i=0}^{n}\left\{\left[l_{2}-1\right.\right. & \left.\left.-i+b\left(i-l_{2}\right)\right] b_{i}-a b_{i-1}+(1-b)(n+1-i) b_{i-2}\right\} x^{n-i} y^{i} \\
& =b l_{2} \sum_{i=0}^{l_{1}}\left(\begin{array}{c}
l_{1} \\
i
\end{array}\right) x^{2 l_{1}+1-2 i} y^{l_{2}+2 i-1}+\left(l_{2} a+L_{0}\right) \sum_{i=0}^{l_{1}}\left(\begin{array}{c}
l_{1} \\
i
\end{array}\right) x^{2 l_{1}-2 i} y^{l_{2}+2 i}
\end{aligned}
$$

where $b_{i}=0$ for $i<0$ and $i>n-1$. From this equation we obtain that

$$
\begin{aligned}
& {\left[l_{2}-1-j+b\left(j-l_{2}\right)\right] b_{j}-a b_{j-1}+(1-b)(n+1-j) b_{j-2}=0} \\
& \quad j=0,1, \ldots, l_{2}-2 \\
& {[-2 i+b(2 i-1)] b_{2 i+l_{2}-1}-a b_{2 i+l_{2}-2}+(1-b)\left(2 l_{1}+2-2 i\right) b_{2 i+l_{2}-3}=b l_{2}\left(\begin{array}{c}
l_{1} \\
i
\end{array}\right)} \\
& {[-2 i-1+2 b i] b_{2 i+l_{2}}-a b_{2 i+l_{2}-1}+(1-b)\left(2 l_{1}+1-2 i\right) b_{2 i+l_{2}-2}=\left(l_{2} a+L_{0}\right)\left(\begin{array}{c}
l_{1} \\
i
\end{array}\right)} \\
& \text { with } i=0,1, \ldots, l_{1} .
\end{aligned}
$$


From the first equation of (51) we can prove that $b_{j}=0$ for $j=0,1, \ldots, l_{2}-2$. From (51) with $i=0,1, \ldots, l_{1}-1$ and its first equation with $i=l_{1}$, working in a similar way to the proof of proposition 10 we can prove that

$b_{l_{2}+2 i-1}=\tilde{\mathcal{B}}_{2 i-1}(a) L_{0}-l_{2}\left(\begin{array}{c}l_{1} \\ i\end{array}\right) \quad b_{l_{2}+2 i}=\tilde{\mathcal{B}}_{2 i}(a) L_{0} \quad i=0,1, \ldots, l_{1}$

where $\tilde{\mathcal{B}}_{k}(a)$ is a polynomial of degree $k$ in $a$ whose coefficients are rational functions in $b$ and $l_{1}$. Using the last equation of (51) with $i=l_{1}$ we get

$$
\left[a \tilde{\mathcal{B}}_{2 l_{1}-1}+(b-1) \tilde{\mathcal{B}}_{2 l_{1}-2}+1\right] L_{0}=0
$$

For every given $b$ and $l_{1}$ there exist at most $2 l_{1}$ values of $a$ for which $a \tilde{\mathcal{B}}_{2 l_{1}-1}+(b-1) \tilde{\mathcal{B}}_{2 l_{1}-2}+1$ is equal to zero. We denote by $\tilde{\gamma}_{l_{1}}$ the set of such $a$. Then if $a \notin \tilde{\gamma}_{l_{1}}$, we have $L_{0}=0$. So for every $b$ satisfying the assumption of the proposition, if $a \notin \cup \tilde{\gamma}_{l_{1}}$ system (7) has no exponential factors.

Case 4: $n>2 l_{1}+l_{2}$. Using the notations of case 2 for $G$ and $G_{n}$, from (46) we get that $\left[x^{2}+a x y+(1-b) y^{2}\right] G_{n x}+\left(b x y+a y^{2}\right) G_{n y}=\left[2 l_{1}(x+a y)+l_{2}(b x+a y)\right] G_{n}$.

Working in a similar way to the previous case we can prove that the coefficients $a_{i}$ in $G_{n}$ satisfy the following equations:

$\left[n-i-2 l_{1}+b\left(i-l_{2}\right)\right] a_{i}+a\left(n-2 l_{1}-l_{2}\right) a_{i-1}+(1-b)(n+2-i) a_{i-2}=0$

with $i=0,1, \ldots, n+1$. Since $b \notin \mathbb{Q}$, from these equations we obtain that $a_{i}=0$. So, $G_{n}=0$. This implies that system (7) has no exponential factors.

Summing up these four cases the proof of the proposition follows.

In this last step we prove that for each $b \notin \mathbb{Q}$, if $a \in \mathbb{R} \backslash\left(\Upsilon^{*} \cup\{0\}\right)$ system (7) is not Darboux integrable.

Suppose that the assumptions of proposition 11 are satisfied. Then, by propositions 10 and 11 we get that system (7) has only the invariant algebraic curves $x^{2}+y^{2}=1$ with cofactor $K_{1}=2(x+a y)$ and $y=1$ with cofactor $K_{2}=b x+a y+a$, and has no exponential factors. It is easy to check that under these assumptions do not exist $\lambda_{1}, \lambda_{2} \in \mathbb{C}$ not all zero such that $\lambda_{1} K_{1}+\lambda_{2} K_{2}=0$ or $\lambda_{1} K_{1}+\lambda_{2} K_{2}=-\operatorname{div}(P, Q)=-(2+b) x-3 a y$, where $P=y(a x-b y+b)+x^{2}+y^{2}-1$ and $Q=b x(y-1)+a\left(y^{2}-1\right)$. Hence, from the Darboux theory of integrability (see, for instance, [9] or [13]) it follows that system (7) is not Darboux integrable. We have finished the proof of theorem 3.

\section{Proof of corollary 4.}

Since $y=1$ is invariant by system (8), after the change of variables

$$
\bar{x}=\frac{x}{y-1} \quad \bar{y}=\frac{y}{y-1} \quad t=\frac{\tau}{y-1}
$$

system (8) becomes the form of system (7), i.e.

$$
\begin{aligned}
& \frac{\mathrm{d} \bar{x}}{\mathrm{~d} \tau}=\bar{y}(a \bar{x}-b \bar{y}+b)+\bar{x}^{2}+\bar{y}^{2}-1=\bar{P}(x, y) \\
& \frac{\mathrm{d} \bar{y}}{\mathrm{~d} \tau}=b \bar{x}(\bar{y}-1)+a\left(\bar{y}^{2}-1\right)=\bar{Q}(x, y) .
\end{aligned}
$$

Let $C(x, y)$ be a polynomial of degree $n$, and set $\bar{C}(\bar{x}, \bar{y})=(\bar{y}-1)^{n} C\left(\frac{\bar{x}}{\bar{y}-1}, \frac{\bar{y}}{\bar{y}-1}\right)$. We claim that if $C(x, y)=0$ is an invariant algebraic curve of system (8) with cofactor $K(x, y)$ 
and $\bar{C} \not \equiv$ constant, then $\bar{C}(\bar{x}, \bar{y})=0$ is an invariant algebraic curve of system (52) with cofactor

$$
\bar{K}=(\bar{y}-1) K\left(\frac{\bar{x}}{\bar{y}-1}, \frac{\bar{y}}{\bar{y}-1}\right)+n \frac{\bar{Q}}{\bar{y}-1} .
$$

Indeed, straightforward calculations show that

$$
\begin{aligned}
\bar{P} \bar{C}_{\bar{x}}+\bar{Q} \bar{C}_{\bar{y}} & =(\bar{y}-1)^{n}\left[\frac{\bar{P}(\bar{y}-1)-\bar{Q} \bar{x}}{(\bar{y}-1)^{2}} C_{x}-\frac{\bar{Q}}{(\bar{y}-1)^{2}} C_{y}+n \frac{\bar{Q}}{\bar{y}-1} C\right] \\
& =(\bar{y}-1)^{n}\left[(\bar{y}-1) P C_{x}+(\bar{y}-1) Q C_{y}+n \frac{\bar{Q}}{\bar{y}-1} C\right] \\
& =(\bar{y}-1)^{n+1} K C+n(\bar{y}-1)^{n} \frac{\bar{Q}}{\bar{y}-1} C=\bar{K} \bar{C}
\end{aligned}
$$

This proves the claim.

Now we claim that if $F(x, y)=\exp \left(\frac{G(x, y)}{H(x, y)}\right)$ is an exponential factor of system (8) with cofactor $L(x, y)$, then

$$
\bar{F}(\bar{x}, \bar{y})=\exp \left(G\left(\frac{\bar{x}}{\bar{y}-1}, \frac{\bar{y}}{\bar{y}-1}\right) / H\left(\frac{\bar{x}}{\bar{y}-1}, \frac{\bar{y}}{\bar{y}-1}\right)\right)
$$

is an exponential factor of system (52) with cofactor $\bar{L}(\bar{x}, \bar{y})=(\bar{y}-1) L\left(\frac{\bar{x}}{\bar{y}-1}, \frac{\bar{y}}{\bar{y}-1}\right)$. In fact, we have

$$
\begin{aligned}
\bar{P} \bar{F}_{\bar{x}}+\bar{Q} \bar{F}_{\bar{y}}= & \exp \left(\frac{G}{H}\right) H^{-2}\left[\left(\frac{\bar{P}(\bar{y}-1)-\bar{Q} \bar{x}}{(\bar{y}-1)^{2}} G_{x}-\frac{\bar{Q}}{(\bar{y}-1)^{2}} G_{y}\right) H\right. \\
& \left.-\left(\frac{\bar{P}(\bar{y}-1)-\bar{Q} \bar{x}}{(\bar{y}-1)^{2}} H_{x}-\frac{\bar{Q}}{(\bar{y}-1)^{2}} H_{y}\right) G\right] \\
= & (\bar{y}-1) \exp \left(\frac{G}{H}\right) H^{-2}\left[\left(P G_{x}+Q G_{y}\right) H-\left(P H_{x}+Q H_{y}\right) G\right] \\
= & (\bar{y}-1)\left(P F_{x}+Q F_{y}\right) \\
= & (\bar{y}-1) L F=(\bar{y}-1) L\left(\frac{\bar{x}}{\bar{y}-1}, \frac{\bar{y}}{\bar{y}-1}\right) \bar{F} .
\end{aligned}
$$

This proves the claim.

From these two claims and the proof of theorem 3 we obtain that there exist values of $a$ and $b$ for which systems (8) and (7) have only two irreducible invariant algebraic curves and no exponential factors. Hence, for such values of $a \neq 0$ and $b$ system (8) is not Darboux integrable. Otherwise, system (7) would have a Darboux integral, in contradiction with theorem 3. Hence, the proof of corollary 4 is complete.

\section{Acknowledgments}

The second and third authors are partially supported by a DGES grant number PB96-1153 and by a CICYT grant number 1999SGR 00349. The fourth author is partially supported by the Ministerio de Educación y Cultura (Spain) with the grant number SB97-50922201 and by NNSFC of China grant number 19901013. He wants to express his thanks to the Centre de Recerca Matemática and to the Departament de Matemàtiques, Universitat Autònoma de Barcelona for its hospitality and support during the period in which this paper was written. 


\section{References}

[1] Andrade R F S and Rauh A 1981 The Lorenz model and the method of Carleman embedding Phys. Lett. A 82 276

[2] Bountis T, Grammaticos B and Ramani A 1989 On the complete and partial integrability of non-Hamiltonian systems Phys. Rep. 180159

[3] Brenig L and Goriely A 1990 Quasimonomial Transformations and Integrability. Partially Integrable Evolution Equations in Physics (Les Houches 1989) (NATO Adv. Sci. Inst. Series. CMath. Phys. Sci. vol 310) (Dordrecht: Kluwer) pp 571-2

[4] Cairó L, Feix M R and Llibre J 1999 Integrability algebraic solutions for planar polynomial differential systems with emphasis on the quadratic systems Resenhas Univ. São Paulo 4 127-61

[5] Cantrijn F and Sarlet W 1981 Generalizations of Noether's theorem in classical mechanics SIAM Rev. 23 467-94

[6] Carleman T 1932 Application de la théorie des équations intégrales linéaires aux systémes d'équations différentielles non linéaires Acta Math. 5963

[7] Chavarriga J, García I A and Giné J 2001 On Lie's symmetries for planar polynomial differential systems Nonlinearity $14863-80$

[8] Chavarriga J, Giacomini H, Gine J and Llibre J 2000 Darbour integrability and the inverse integrating factor Preprint

[9] Chavarriga J, Llibre J and Sotomayor J 1997 Algebraic solutions for polynomial vector fields with emphasis in the quadratic case Expositions Math. 15 161-73

[10] Christopher C 1994 Invariant algebraic curves and conditions for a center Proc. R. Soc. Edin. A 124 1209-29

[11] Christopher C 1993 Polynomial systems with invariant algebraic curves Preprint

[12] Kooij R and Christopher C 1993 Algebraic invariant curves and the integrability of polynomial systems Appl. Math. Lett. 6 51-53

[13] Christopher C and Llibre J 2000 Integrability via invariant algebraic curves for planar polynomial differential systems Ann. Differ. Equ. 14 5-19

[14] Darboux G 1878 Mémoire sur les équations différentielles algébriques du premier ordre et du premier degré (Mélanges) Bull. Sci. Math. 2 60-96, 123-44, 151-200

[15] Fulton W 1969 Algebraic Curves (New York: Benjamin)

[16] Giacomini H J, Repetto C E and Zandron O P 1991 Integrals of motion of three-dimensional non-Hamiltonian dynamical systems J. Phys. A: Math. Gen. 24 4567-74

[17] Hietarinta J 1987 Direct methods for the search of the second invariant Phys. Rep. 147 87-154

[18] Jouanolou J P 1979 Equations de Pfaff algébriques (Lectures Notes in Mathematics vol 708) (Berlin: Springer)

[19] Lax P D 1968 Integrals of nonlinear equations of evolution and solitary waves Commun. Pure Appl. Math. 21 467-490

[20] Christopher C, Llibre J and Pereira J V 2000 Multiplicity of invariant algebaric curves and Darboux integrability Preprint

[21] Llibre J, Pérez del Río J S and Rodríguez J A 2000 Phase portraits of a new class of integrable quadratic vector fields Dyn. Contin. Discrete Impuls. Syst. 7 595-616

[22] Maciejewski A, Moulin Ollagnier J, Nowicki A and Strecyn J-M 2000 Around Jouanolou non-integrability theorem Indagationes Math. 11 239-54

[23] Olver P J 1986 Applications of Lie groups to Differential Equations (New York: Springer)

[24] Poincaré H 1891 Sur l'intégration des équations différentielles du premier ordre et du premier degré I and II Rendiconti del Circolo Matematico di Palermo 5 161-91

Poincaré H 1897 Rendiconti del Circolo Matematico di Palermo 11 193-239

[25] Prelle M J and Singer M F 1983 Elementary first integrals of differential equations Trans. Am. Math. Soc. 279 613-36

[26] Singer M F 1992 Liouvillian first integrals of differential equations Trans. Am. Math. Soc. 333 673-88

[27] Strelcyn J M and Wojciechowski S 1988 A method of finding integrals for three-dimensional dynamical systems Phys. Lett. A 133 207-12

[28] Weil J A 1995 Constant et polynómes de Darboux en algébre différentielle: applications aux systémes différentiels linéaires $P h D$ Thesis Ecole Polytecnique

[29] Zholadek H 1992 The solution of the center-focus problem Preprint

[30] Zholadek H 1995 On algebraic solutions of algebraic Pfaff equations Stud. Math. 114 117-26 